\title{
Proposed treatment for adolescent psychosis. 2: Bipolar illness
}

\author{
Andrew Clark
}

Bipolar illnesses are typically considered as disorders primarily occurring in adult life, but a substantial number have their onset during the teenage years. Any acute illness in the teenage years affects social, emotional and educational development disproportionately, as it may prevent the normal transitions and learning experiences inherent in adolescence. Additionally, there is some evidence for a lack of early recognition or for misdiagnosis in early-onset cases of psychiatric disorders in general and the psychotic disorders in particular, with consequent increased suffering and impairment of functioning. Finally, bipolar affective disorders rarely present with one single episode followed by full and permanent recovery, but are usually illnesses characterised by a relapsing and remitting course through adult life. Prompt and effective recognition and treatment may therefore not merely reduce immediate impact but may also set up patterns of understanding and treatment compliance with longer-term implications. In this paper, I consider the particular features of bipolar affective disorder in adolescence and to describe a practical framework for its management. In view of the relative lack of age-specific research findings, particularly in respect of treatment effectiveness, I draw heavily upon clinical practice and extrapolation from studies carried out among adult sufferers.

\section{Diagnostic criteria}

The diagnostic criteria for a diagnosis of a bipolar affective disorder are broadly the same, irrespective

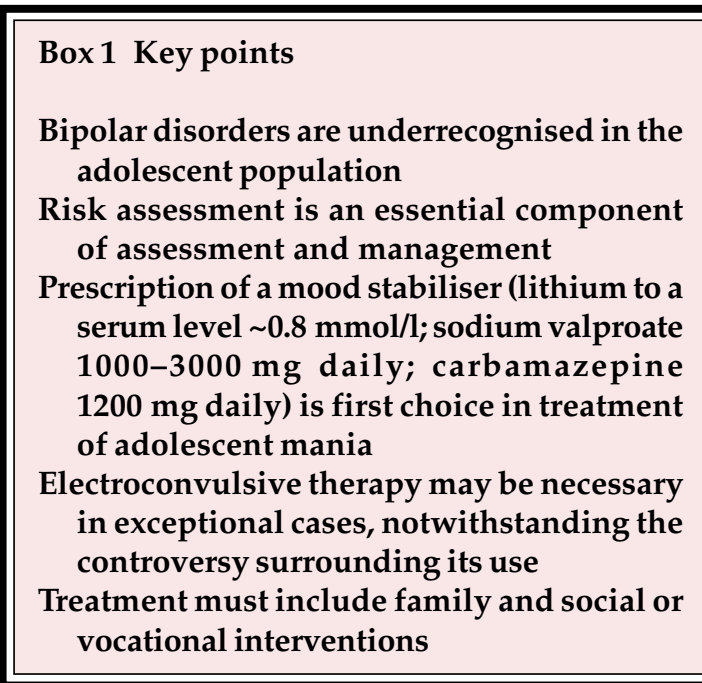

of age of onset. The core feature is repeated (i.e. at least two) episodes of elevated or depressed mood. Both ICD-10 (World Health Organization, 1992) and DSM-IV (American Psychiatric Association, 1994) discriminate between lesser degrees of mood elevation (hypomania) and more severe degrees (mania with or without psychotic symptoms). Accompanying features are increased energy and activity, increased talkativeness and increased sense of wellbeing and physical prowess. With more severe illnesses, inflated self-esteem and grandiosity may reach delusional proportions. In either classificatory system, the illness has to present for at least a week for a diagnosis of mania, although this restriction does not apply to hypomania. Depressive episodes are similarly divided by severity and by presence or

Andrew Clark has been a senior lecturer in adolescent psychiatry in Manchester since 1997 (McGuinness Unit, Mental Health Services of Salford NHS Trust, Bury New Road, Prestwich, Manchester, M25 3BL; e-mail: andrew.clark@man.ac.uk) and was previously a consultant in adolescent psychiatry in the West Midlands region from 1990 to 1997 . He has particular interest in the assessment and treatment of young people with psychotic disorders. 
absence of psychotic symptoms. It is possible also to have a clinical presentation in which features of hypomania or mania coexist with features of a depressive episode-such episodes are defined as a mixed affective episode.

DSM-IV additionally recognises as separate disorders both rapid cycling mood disorder, where episodes of hypomania, mania or depression occur at least four times in a 12-month period, and cyclothymic disorder, which is characterised by symptoms of lesser severity but present for much of the time (with no more than two consecutive months symptom-free). Cyclothymia is very similarly described within ICD-10, albeit without a criterion regarding duration. ICD-10 does not, however, recognise rapid cycling mood disorders as a separate entity, but rather contains them under the more general rubric of bipolar affective disorder.

\section{Epidemiology}

There are considerable grounds for believing that bipolar illness is underrecognised in childhood and adolescence. While there is some dispute about its existence and nature prepubertally, and the degree to which some cases may overlap with conditions such as attention-deficit disorders, it seems clear that the disorder can present in adolescence in a manner that is indistinguishable in natural history from cases with later onset. Surveys among adults with established bipolar illnesses have suggested that a significant minority (20-40\%) date the onset of their illness to age 20 years or younger. In a community survey of American teenagers, Lewinsohn et al (1995) found a prevalence for bipolar affective disorders of $1 \%$, with an approximately equal ratio between boys and girls.

\section{Clinical presentations}

Dependent upon the phase of the disorder, the young person may exhibit symptoms of depression, mania or hypomania, a mixed state, or indeed no psychiatric symptoms at all. A depressive phase is likely to be characterised by apathy, social withdrawal, pessimism and a loss of interests and pleasure. This may result in the teenager becoming more truculent and irritable, spending more time alone, performing less well in school and in expressing miserable, hopeless or suicidal ideas. In contrast, the teenager with hypomania or mania is likely to be overactive, full of energy, full of self-belief and challenging of authority and with overinflated ideas of ability and prowess. He or she may express unrealistic ambitions or expectations to become a rock star or sporting legend, irrespective of any actual level of talent or ability. Irritability, particularly when grandiose beliefs or plans are challenged, is frequently a feature, as may be exasperation at the perceived shortcomings of others. These can lead to aggressive or violent threats or even actual assault. School performance is likely to deteriorate owing to poor concentration and a failure to complete any of the work started. Excessive spending can be a problem, particularly if the teenager can gain access to parental credit cards and use these over the telephone. Sexual disinhibition and risk-taking may also be a feature of a manic or hypomanic episode and may be a focus of considerable regret and embarrassment when subsequently recovered. Mixed affective states contain symptoms of both mood poles occurring together over a period of at least 2 weeks, for example, coexistence of low mood with overactivity and pressure of speech or of elevated mood and grandiosity with agitation, loss of energy, drive and libido. Between episodes of disturbed mood, most adolescents will be asymptomatic, although there are suggestions that they may be more prone to rapid cycling disorders and therefore that periods of 'wellness' may be shorter than in adults.

\section{Differential diagnosis}

In most cases, the diagnosis of a bipolar disorder will be considered only with the first occurrence of a manic episode. However, in a number there may have been previous depressive episodes (which may or may not have received formal treatment in their own right) and in others a pattern of mood fluctuation of a cyclothymic nature. More severe cases are likely to pose fewer but different diagnostic challenges than less severe cases. In the more severe cases, particularly those with psychotic symptoms, the uncertainty will be in discriminating between manic disorder, schizophrenia-like disorders (including schizoaffective disorders) and organic or substance-induced disorders, rather than in the recognition of an illness process itself. In lesser degrees of illness, however, there may be considerable difficulty in discriminating milder degrees of hypomania from conduct or oppositional-defiant type symptoms. Minor degrees of mood elevation, self-importance, overactivity and questioning of adults can easily be attributed to conduct disorder or even to adolescence itself rather than consideration of an illness. This is particularly so when there is a pre-existing history of conduct disturbance or 
symptoms similar to those of attention-deficit disorder. Eliciting evidence of periodicity and significant sustained variation over time is the main means of attempting to clarify the diagnosis, but considerable uncertainty can none the less remain in some cases.

Distinguishing an acute manic state from a schizophrenic disorder appears particularly problematic. In some cases, a diagnosis of schizoaffective disorder may be appropriate, but this does require simultaneous experience of symptoms both of schizophrenia and of a manic, depressive or mixed affective state. However, in other cases of diagnostic uncertainty the psychotic beliefs may appear bizarre and extremely persecutory, any hallucinations may appear mood-incongruent and the mood state may show considerable lability. This may be particularly so in a mixed affective state. Additionally, self-care and social functioning may show a marked deterioration from premorbid levels. These features can lead to high rates of misdiagnosis of schizophrenia (McClellan et al, 1993). While early diagnosis can facilitate treatment planning, inform prognosis and can give both the young person and the family a language for discussion, it needs to be as accurate as possible. In some instances, it may be most appropriate to avoid an early definitive diagnosis and instead share the diagnostic uncertainty with the family and await clarification as the clinical picture evolves. Pointers towards a primary diagnosis of schizophrenia are those of a family history of schizophrenia, an insidious onset and the presence of significant thought disorder. The assessment and management of schizophrenia and related psychoses in adolescence are given fuller consideration in Hollis (2000) and Clark (2001).

Acute or chronic organic states presenting clinically as hypomania or mania (so-called secondary mania) are rare but do occur in association with both substance misuse and a wide variety of physical illnesses. It is important to keep an index of suspicion of physical disorder and to assess and investigate possible physical causes appropriately (see below and Box 2).

Box 2 Physical assessment

Carry out a physical examination in all cases Obtain a full blood count, urea and electrolytes, liver- and thyroid-function tests and urinary drug screen in all cases

Obtain electroencephalograms and computer tomography or magnetic resonance imaging scans only where specific indications exist (these may include parental anxiety)

\section{Assessment}

Assessment should include a history of recent behaviours, social functioning and emotional state from both the young person and a carer. Reports from school or college are extremely helpful in amplifying both aspects of social functioning and in identifying any recent impairment in cognitive performance (especially attention or concentration). A detailed developmental history may reveal evidence of previous mood disturbance, particularly of an episodic or cyclical nature, pointing towards previous lesser degrees of illness having been present for some time, albeit unrecognised as such. A family history of affective disorder is an additional pointer towards diagnosis, although this should not lessen the need for accurate assessment of core symptoms.

This initial assessment should also include some form of risk assessment. This should have a particular focus upon the potential for suicide, the risks to self from impulsive or excessive risk-taking behaviour (e.g. attempting to drive the family car at the age of 14 years), and the risks to others from irritability, aggression or impulsive or dangerous behaviours. This will need to take account of current mental state, including level of insight and attitude to treatment, recent behaviour during this period of illness, behaviours during any previous episodes of illness and any element of interpersonal risk unassociated with illness. Its conclusions will inform subsequent decision-making about how and where to treat the young person and about any special precautions necessary. Assessment of risk is not static and any conclusions and consequent measures will need regular review throughout the course of the illness.

In most young people presenting with signs and symptoms of an acute affective disorder, the physical examination will be unremarkable. It none the less remains an essential part of initial assessment, as a number of acute general medical conditions (e.g. cerebral tumour, hyperthyroidism, porphyria and prescribed and non-prescribed medication) can, albeit rarely, produce manic symptoms. A full blood count, urea and electrolytes, tests of liver and thyroid function, and a urinary drug screen should all be performed, both as part of diagnostic assessment and prior to instituting pharmacotherapy. Electroencephalogram and computer tomography or magnetic resonance imaging scans are often requested but rarely produce clinically significant findings in the absence of positive indicators of organicity in the history, mental state or physical examination. They may, however, be reassuring to both the patient and the family, and this may in itself be sufficient reason for their taking place. 


\section{Management and treatment}

Management and treatment needs to include the current depressive episode and its recovery, the current manic episode and its recovery and the period of wellness between acute episodes of illness. In each phase, it is also necessary to consider pharmacological, psychological and social interventions.

\section{Management of a depressive episode}

The management of depressive episodes in adolescence is well described in detail elsewhere. In essence, mild to moderate depressive episodes are best treated psychologically, but more severe episodes may require pharmacological treatment (Harrington et al, 1998a). Randomised controlled trials of tricyclic antidepressants in treatment of adolescent depressive disorders have consistently failed to demonstrate any therapeutic benefit and these should therefore usually be avoided. Emslie $e t$ al (1997) have, however, demonstrated the efficacy of fluoxetine in reduction of depressive symptoms in teenagers with depression and this (or another selective serotonin reuptake inhibitor (SSRI)) should be the drug treatment of choice. Drug treatments are rarely given in isolation but rather usually in combination with some form of individual psychological intervention, either primarily supportive or more cognitively based.

A number of young people with an emergent bipolar disorder initially present with recurrent unipolar depressive episodes and are initially treated within the above guidelines. Up to $20-30 \%$ of patients presenting with early-onset depressive disorder may eventually develop a bipolar disorder. Clinical features most associated with this shift appear to be rapid onset, symptoms of psychomotor retardation or psychosis, a family history of affective disorder and a history of elevated mood states on treatment with antidepressants.

Treatment of a depressive episode in the context of established bipolar disorder is more complex. In some instances, the depression is immediately following or in the recovery phase of a manic episode. It may be difficult in these cases to determine whether it is low mood occurring as part of the illness process or low mood in response to developing insight as to the seriousness of the illness and/ or the consequences of acts during its acute phase. Many of these episodes resolve upon continuation of the combination of antimanic or mood-stabilising drug treatment and supportive psychotherapies and do not require specific antidepressant drug treatment in their own right.

If pharmacotherapy is necessary, then there is a risk of precipitating a further manic episode. This seems to be present irrespective of which class of antidepressant is chosen. Initially, therefore, prescription of or increase in the dosage of mood stabiliser may be the first choice. If low mood persists and there is felt to be little alternative to prescription of an antidepressant, then it seems sensible that this should only be in combination with a mood stabiliser rather than in isolation, notwithstanding the increased hazards of polypharmacy. Again, an SSRI should be preferred to a tricyclic antidepressant on grounds of both safety and efficacy.

\section{Management of a manic episode}

The management of a young person with an acute manic or hypomanic episode poses particular challenges. Recognition may be delayed as symptoms are initially attributed to a drive for increased independence or autonomy or to sexual experimentation or to oppositional or conduct disturbance. With increasing severity of illness, recognition may be easier, but increasing levels of risk to self or others and decreasing levels of insight may both complicate treatment.

Engagement may prove particularly problematic if the young person has little or no insight into the condition and is therefore reluctant to consider medication. Persuasion and close supervision may be necessary to ensure compliance. In more severe cases, admission to hospital may be necessary to deliver the level of care required or to ensure the safety of the sufferer or others. Ideally, this should be into an age-appropriate adolescent psychiatric unit, but there are frequently bed shortages and other solutions may need to be sought (including admission to adult in-patient facilities) (Cotgrove \& Gowers, 1999). However, the National Service Framework (Department of Health, 1999) for the National Health Service in England and Wales, although focused upon working-age adults, does now require health authorities to make explicit plans for provision of services for teenagers requiring acute psychiatric admission. In particularly severe cases, it may even be necessary to consider compulsory admission against the teenager's wishes. This could be achieved either through the use of the mental health legislation or through reliance upon parental consents on behalf of a minor. The law regarding a young person's capacity and ability to consent to or refuse treatment is complex and contradictory and each case needs careful individual consideration of the most appropriate 
legal mandate. Bailey \& Harbour (1998) give a helpful overview of the current legal position on consent in England and Wales.

There are two alternative strategies for drug treatment of a manic episode - prescription of a mood stabiliser or prescription of a major tranquilliser or antipsychotic drug. Lithium preparations used to be the only choice as a mood stabiliser and they do have the virtue of one age-specific randomised controlled trial in bipolar disorder to support their use, but carbamazepine and sodium valproate are also increasingly being considered as possible firstline treatments. While there is little robust research evidence to guide the clinician, they do appear to be at least as effective and better tolerated, with less need for intensive monitoring. This may be a particular benefit in young people or families where there may be difficulties in treatment compliance and adherence. None the less, they carry significant risks of hepatotoxicity, blood dyscrasis and, in the case of valproate, possibly polycystic ovaries (Ryan et al, 1999). Additionally, prescribers should be aware that such usage is beyond the product licence for both drugs and this should be explicitly discussed with the young person and the family (notwithstanding that their usage is similarly beyond product licence in adulthood, except for carbamazepine in rapid cycling mood disorders). Other anticonvulsants, such as gabapentin and lamotrigine, have also been suggested as possessing moodstabilising properties, although experience in their usage remains extremely limited in child and adolescent mental health.

There is little to guide the choice between major tranquilliser and mood stabiliser in the acute phase, although the medium-term aim should be use of a mood stabiliser as maintenance treatment. Both groups of drugs have significant side-effects, in both short- and long-term usage. The newer atypical antipsychotics, such as risperidone or olanzapine, may have a reduced and different side-effect profile than older agents such as haloperidol, but are still far from devoid of side-effects. They also have relatively age-specific efficacy and safety data (Campbell et al, 1999). There are similarly relatively few data concerning the long-term usage of lithium in children and adolescents, but carbamazepine and valproate are both well established in the treatment of epilepsy, and consequently valproate is perhaps increasingly becoming the drug of first choice in the treatment of adolescent mania (Ryan et al, 1999). In rapid cycling or mixed disorders, there is some evidence from studies of adult sufferers that lithium is relatively ineffective and that here carbamazepine and valproate are clearly to be preferred.

While the therapeutic levels for lithium in treatment of bipolar disorders are now well established at around $0.8 \mathrm{mmol} / 1$, there is little such agreement for carbamazepine or valproate. Recommended levels and dosages therefore tend to be similar to those guiding their use as anticonvulsants - for valproate, between 1000 and $3000 \mathrm{mg}$ daily and for carbamazepine up to $1200 \mathrm{mg}$ daily (American Academy of Child and Adolescent Psychiatry, 1997). In both instances, the dosage should be built up gradually and should be accompanied by periodic monitoring of serum levels. Adjunctive treatment with an antipsychotic or, for preference, a benzodiazepine may be useful in the acute phase if additional sedation is required (e.g. lorazepam 2$4 \mathrm{mg}$ daily). For those few patients who remain ill despite adequate dosages of a single mood stabiliser over a period of 4-6 weeks, combination therapy may be necessary, and a combination of lithium and valproate would seem to offer most benefits and fewest risks (Freeman \& Stoll, 1998).

Very rarely, young people present with a manic or depressive disorder of such a severity and lifethreatening nature that a very rapid response to treatment is necessary. On these occasions, electroconvulsive therapy (ECT) should be considered, notwithstanding the patient's youth. Although data are sparse, and certainly there are no age-specific randomised controlled trial findings to draw upon, there is some limited evidence for its prescription to teenagers with severe affective psychoses from case reports of its apparent efficacy and safety (Walter $e t$ $a l, 1999 a)$ and from patient questionnaires regarding its patient acceptability (Walter et al, 1999b). None the less, given the controversies surrounding the use of ECT, it is probably prudent to obtain an independent second opinion before proceeding with its prescription and administration. Indeed, Freeman (1995) advocates obtaining independent opinions from both a child and adolescent psychiatrist and an adult psychiatrist when considering ECT treatment of anyone under 16 years of age. This should be in addition to any further opinion necessary for mandatory treatment under mental health legislation. Walter et al (1999b) also provide a useful factsheet for use with young people and their families when ECT is being considered.

\section{Treatment between acute episodes}

This needs to be divided into continuation of treatment after an acute episode for an adequate length of time in order to reduce risk of early relapse, and maintenance or prophylactic treatment between episodes. After any acute episode of affective illness necessitating drug treatment, then mood stabilising treatment should be continued for at least 6-9 months post-recovery. Cessation should be negotiated with 
the young person and the family and should be by gradual reduction, under close supervision and ideally at a time of stability in the young person's life. This is clearly a challenge, given that adolescence is a time of many changes and transitions physically, socially and educationally.

Episodes of affective illness, particularly of a manic nature, can exert a devastating effect upon a young person's family, social and educational life and prevention of further bouts of illness during the formative years of adolescence is a major treatment goal. This means that after even one episode of illness there may be an argument for continuing prescription of mood stabilising medication beyond that necessary for mere early-relapse prevention. This is particularly so if there are grounds for believing an individual to be at higher risk of subsequent episodes (e.g. family history of affective disorder). This needs careful discussion with the young person and his or her parents, although often they may be more anxious about the risks of relapse upon stopping medication than about any disadvantages inherent in long-term treatment.

\section{Psychological and psychosocial interventions}

Any treatment package needs to be multi-modal, encompassing not merely pharmacotherapy but also individual and family psychological interventions and social and vocational measures. While the role of individual and family interventions has been formally evaluated in child and adolescent depressive disorder (Harrington et al, 1998b), this is not so for bipolar disorders. Extrapolation from adult studies would clearly suggest that such interventions should both enhance compliance and coping and prevent relapse (Scott, 1999). Initially, individual psychological intervention is likely to be more information-giving and supportive in nature, with an emphasis on the illness and its treatments. More formal cognitive strategies may be introduced as the acute phase resolves, using approaches such as motivational interviewing to encourage regular medication. Family intervention also focuses initially upon support and information-giving, but is likely to move towards psycho-educational approaches, with the aim of reducing high levels of expressed emotion if present. Psychosocial interventions need to involve educational and vocational aspects, with school and college liaison often being an important part in supporting a young person's rehabilitation.

Voluntary and self-help organisations can be useful sources of support and written information for young people, their siblings and their parents
Box 3 Useful addresses

Young Minds: 2nd Floor, 102 Clerkenwell Road, London EC1M 5SA

MIND: Granta House, 15-19 Broadway, Stratford, London E15 4BQ

Manic Depression Fellowship: 6-10 High Street, Kingston-upon-Thames KT1 1EY

(see Box 3). A number of other organisations also publish factsheets regarding bipolar affective disorder and its treatment that can be extremely useful in giving parents and young people accessible information. Examples of these include the American Academy of Child and Adolescent Psychiatry and the Royal College of Psychiatrists (factsheets from both may be downloaded from the internet) and the United Kingdom Psychiatric Pharmacy Group.

\section{Outcome}

While the short-term outcome for any individual episode of illness is relatively good, this may not be the case in the longer term. Follow-up studies of those young people admitted to hospital with an acute episode (who may by definition have more severe illnesses) suggest that the longer-term outcome is frequently one of continuing episodes of illness and hospital admissions, with marked social impairment (Gillberg et al, 1993; McClellan et al, 1993; Cawthron et al, 1994). This emphasises the need for good psycho-educational intervention with both the young person and his or her family (particularly around treatment compliance and relapse recognition) and for careful preparation and planning of any transition from child and adolescent to adult mental health services. Treatment planning should take place within a Care Programme Approach framework (even though its provisions do not formally apply to the under-16 age group), and wherever possible transitions should be staggered so that the young person and the family are always in contact with at least one professional familiar with their care.

\section{References}

\footnotetext{
*American Academy of Child and Adolescent Psychiatry (1997) Practice parameters for the assessment and treatment of children and adolescents with bipolar disorder.
} 
Journal of the American Academy of Child and Adolescent Psychiatry, 36 (suppl. 10), 157S-176S.

American Psychiatric Association (1994) Diagnostic and Statistical Manual of Mental Disorders (4th edn) (DSM-IV). Washington, DC: APA.

*Bailey, S. \& Harbour, A. (1998) The law and a child's consent to treatment (England and Wales). Child Psychology and Psychiatry Review, 4, 1-5.

*Campbell, M., Rapoport, J. L. \& Simpson, G. M. (1999) Antipsychotics in children and adolescents. Journal of the American Academy of Child and Adolescent Psychiatry, 38 537-545.

Cawthron, P., James, A., Dell, J., et al (1994) Adolescent onset psychosis. A clinical and outcome study. Journal of Child Psychology and Psychiatry, 35, 1321-1332.

Clark, A.F. (2001) Proposed treatment for adolescent psychosis. 1: Schizophrenia and schizophrenia-like psychoses. Advances in Psychiatric Treatment, 7, 16-23.

Cotgrove, A. J. \& Gowers, S. G. (1999) Use of an adolescent in-patient unit. Advances in Psychiatric Treatment, 5, 192199.

Department of Health (1999) National Service Framework for Mental Health. London: Department of Health.

Emslie, G. J., Rush, A. J., Weinberg, W. A., et al (1997) A double blind randomized placebo controlled trial of fluoxetine in children and adolescents with depression. Archives of General Psychiatry, 54, 1031-1037.

Freeman, C. P. (1995) ECT in those under 18 years old. In The ECT Handbook (ed. C.P. Freeman), pp. 18-21). London: Royal College of Psychiatrists.

Freeman, M. P. \& Stoll, A. L. (1998) Mood stabilizer combinations: a review of safety and efficacy. American Journal of Psychiatry, 155, 12-21.

Gillberg, I. C., Hellgren, L. \& Gillberg, C. (1993) Psychotic disorders diagnosed in adolescence. Outcome at 30 years. Journal of Child Psychology and Psychiatry, 34, 11731186.

Harrington, R., Whittaker, J., Shoebridge, P., et al (1998a) Systematic review of efficacy of cognitive behaviour therapies in childhood and adolescent depressive disorder. British Medical Journal, 316, 1559-1563.

_ _ _ \& — (1998b) Psychological treatment of depression in children and adolescents. A review of treatment research. British Journal of Psychiatry, 173, 291-298.

Hollis, C. (2000) Adolescent schizophrenia. Advances in Psychiatric Treatment, 6, 83-92.

Lewinsohn, P. M., Klein, D. N. \& Seeley, J. R. (1995) Bipolar disorders in a community sample of older adolescents: prevalence, phenomenology, comorbidity and course. Journal of the American Academy of Child and Adolescent Psychiatry, 34, 454-463.

McClellan, J. M., Werry, J. S. \& Ham, M. (1993) A follow-up study of early onset psychosis: comparison between outcome diagnoses of schizophrenia, mood disorders and personality disorders. Journal of Autism and Developmental Disorders, 23, 243-262.

* Ryan, N. D., Bhatara, V. S. \& Perel, J. M. (1999) Mood stabilizers in children and adolescents. Journal of the American Academy of Child and Adolescent Psychiatry, 38, $529-536$.

Scott, J. (1999) Cognitive and behavioural approaches to medication adherence. Advances in Psychiatric Treatment, 5, 338-345.

*Walter, G., Rey, J. M. \& Mitchell, P. B. (1999a) Practitioner review: electroconvulsive therapy in adolescents. Journal of Child Psychology and Psychiatry, 40, 325-334.

—, Koster, K. \& Rey, J. M. (1999b) Electroconvulsive therapy in adolescents: experience, knowledge, and attitudes of recipients. Journal of the American Academy of Child and Adolescent Psychiatry, 38, 594-599.

World Health Organization (1992) The ICD-10 Classification of Mental and Behavioural Disorders: Clinical Descriptions and Diagnostic Guidelines. Geneva: World Health Organization.

* indicates articles of particular interest

\section{Multiple choice questions}

1. Bipolar disorder in adolescents:

a has a better long-term outcome than in adults

b occurs in up to $1 \%$ of the general adolescent population

c is probably underrecognised

d may initially be misdiagnosed as conduct disorder

e is unlikely to recur in adulthood.

2. Depressive symptoms in the context of established bipolar disorder:

a should be treated with a tricyclic antidepressant

b may be a response to developing insight about the illness or its consequences

c may not respond to psychotherapeutic measures alone

d carry a significant risk of suicide

e can coexist with symptoms more suggestive of a manic episode.

3. Physical treatments used in treatment of bipolar disorder in adolescence:

a are underpinned by a robust research evidence base

b may include ECT

c should not be given in isolation from psychological interventions

d should be considered only when psychological interventions have failed

e may themselves precipitate a manic or depressive episode.

4. When used as mood stabilisers, carbamazepine and sodium valproate:

a do not require monitoring of serum levels

b may be prescribed in combination with an SSRI

c are to be preferred to lithium in rapid cycling disorders

$\mathrm{d}$ may be augmented by benzodiazepines or antipsychotics for extra sedation

e may be associated with long-term side-effects.

\section{MCQ answers}

$\begin{array}{lllll}\text { 1 } & & \text { 2 } & 3 & 4 \\ \text { a F } & \text { a F } & \text { a F } & \text { a F } \\ \text { b T } & \text { b T } & \text { b T } & \text { b T } \\ \text { c T } & \text { c T } & \text { c T } & \text { c T } \\ \text { d T } & \text { d T } & \text { d F } & \text { d T } \\ \text { e F } & \text { e T } & \text { e T } & \text { e T }\end{array}$

